\title{
A multifaceted 'omics' approach for addressing the challenge of antimicrobial resistance
}

\author{
Asi Cohen', Louis Bont ${ }^{2}$, Dan Engelhard ${ }^{3}$, Edward Moore ${ }^{4}$, David Fernández ${ }^{5}$, \\ Racheli Kreisberg-Greenblatt ${ }^{6}$, Kfir Oved ${ }^{1}$, Eran Eden' ${ }^{1}$ \& John P Hays ${ }^{*, 7}$
}

\begin{abstract}
The inappropriate use of antibiotics has severe global health and economic consequences, including the emergence of antibiotic-resistant bacteria. A major driver of antibiotic misuse is the inability to accurately distinguish between bacterial and viral infections based on currently available diagnostic solutions. A multifaceted 'omics' approach that integrates personalized patient data such as genetic predisposition to infections (genomics), natural microbiota composition and immune response to infection (proteomics and transcriptomics) together with comprehensive pathogen profiling has the potential to help physicians improve their antimicrobial prescribing practices. In this respect, the EU has funded a multidisciplinary project (TAILORED-Treatment) that will develop novel omics-based personalized treatment schemes that have the potential to reduce antibiotic consumption, and help limiting the spread of antibiotic resistance.
\end{abstract}

\section{The antibiotic-resistance crisis}

The treatment of infectious disease using antibiotics has been one of the most important advances in modern healthcare, saving millions of lives since their discovery and widespread use. Despite their immense contribution to global healthcare, the CDC recently reported that 'up to $50 \%$ of all the antibiotics prescribed for people are not needed or are not optimally effective as prescribed' [1]. Antibiotic overuse typically stems from prescribing these drugs to treat nonbacterial diseases (mostly viral infections) for which they are ineffective. For example, in the USA alone, over 60 million annual cases of viral influenza are prescribed unnecessary antibiotic therapy [2]. Antibiotic misuse has severe health and economic outcomes (Figure 1). Overprescription of antibiotics may cause preventable adverse events such as allergic reactions, intestinal yeast infection and antibiotic-associated diarrhea [3]. These preventable adverse events may impact patient care and result in lengthy hospitalization. In fact, antibiotics are the most common cause of emergency department visits for adverse events in children under the age of 18 years [1]. Conversely, delayed or no antibiotic treatment in cases of bacterial disease is also common (24-40\% of all bacterial infections) [4-7]. While this may reduce the risk of antibiotic-related adverse events, such practices can lead to disease-related complications resulting in increased rates of morbidity and mortality [8-10].

'MeMed Diagnostics, Tirat Carmel, Israel

2Department of Pediatric Infectious Diseases \& Immunology \& Laboratory of Translational Immunology, Wilhelmina Children's

Hospital, University Medical Center Utrecht, Utrecht, The Netherlands

${ }_{3}^{3}$ Pediatric Department, Pediatric Infectious Disease Unit, Hadassah-Hebrew University Medical Center, Jerusalem, Israe

${ }^{4}$ University of Gothenburg, Gothenburg, Sweden

${ }^{5}$ Noray Bioinformatics, Derio, Spain

6/bexperts Ltd, Ra'anana, Israel

7Department of Medical Microbiology \& Infectious Diseases, Erasmus University Medical Center (Erasmus MC), Rotterdam,

The Netherlands

*Author for correspondence: j.hays@erasmusmc.nl

\section{KEYWORDS}

- antibiotic resistance

- bioinformatics • genomics

- mass spectrometry

- microbiota - proteomics

- transcriptomics 


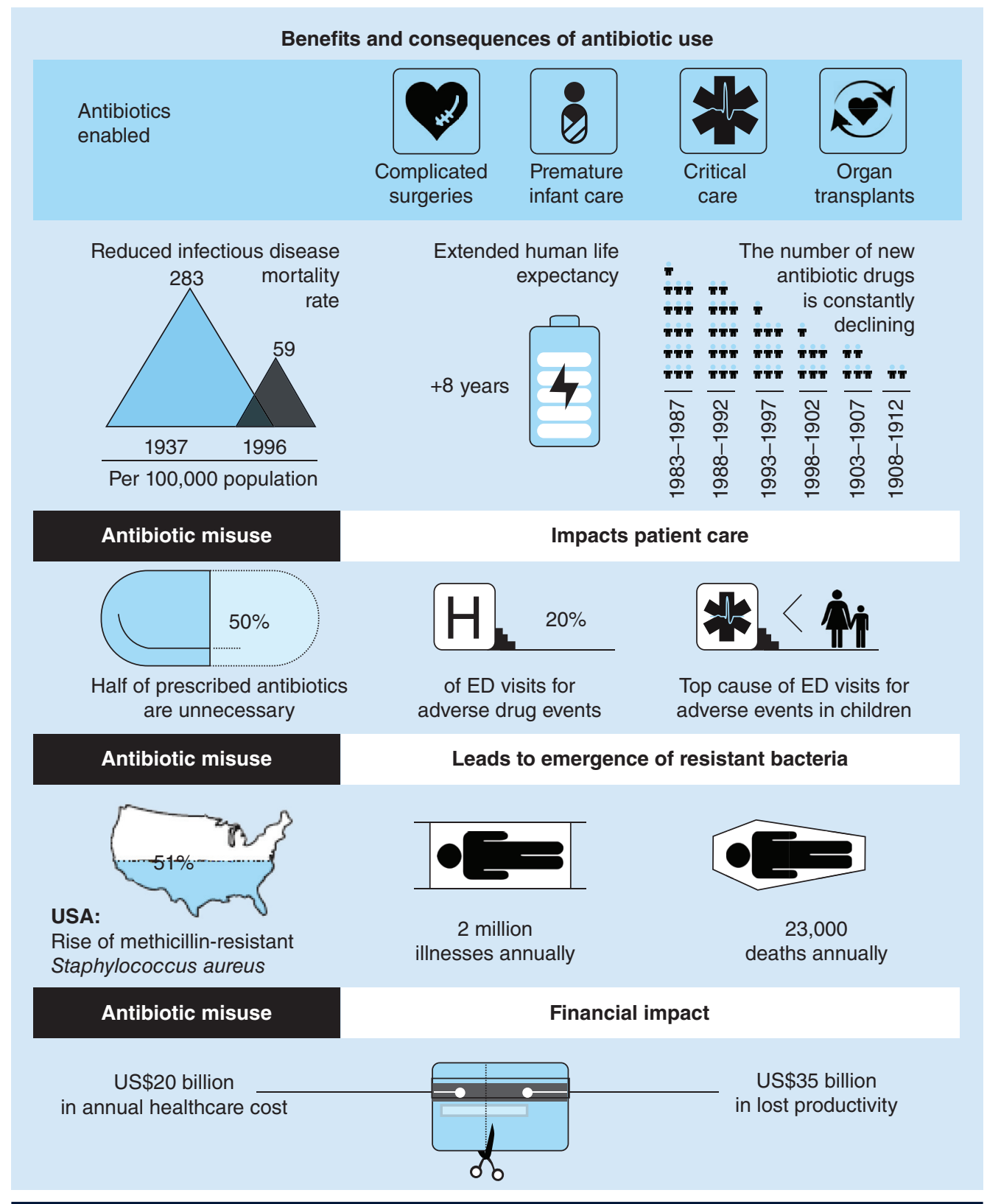

Figure 1. Despite the immense contribution of antibiotics to global healthcare, antibiotic misuse has severe health and economic consequences.

ED: Emergency department.

Data taken from $[1,11-13]$.

One of the most alarming consequences of antibiotic overuse is the emergence and spread of multidrug-resistant bacteria. Resistance of microbial pathogens to antibiotics is increasing worldwide at an accelerating rate $[1-2,14-15]$, with a concomitant increase in morbidity and mortality associated with infections caused by antibiotic-resistant pathogens [1]. At least 2 million people are infected with antibioticresistant bacteria each year in the USA alone, and at least 23,000 people die as a direct result of these infections [1]. In the EU, an estimated 400,000 patients present with resistant bacterial strains each year, of which 25,000 patients die [16]. Consequently, the WHO has warned that therapeutic coverage will be insufficient within 10 years, putting the world at risk of entering a 'postantibiotic era', in which antibiotics will no longer be effective against infectious diseases [17]. The CDC considers this 
phenomenon 'one of the world's most pressing health problems in the 21st century' $[2,18]$.

\section{The diagnostic gap}

Antibiotic overuse in hospitals and outpatient settings contributes significantly to the rising prevalence of antibiotic resistance $[2,11,14]$. At the heart of this problem is the challenge of accurately distinguishing between bacterial infections (which warrant antibiotic therapy) and viral infections (for which antibiotic treatment is generally not required). This diagnostic gap is driven by the inability of current diagnostic tools to provide rapid and accurate information regarding the etiological basis of an infection.

\section{- Technical limitations of current diagnostics}

Conventional diagnostic approaches depend on the cultivation of infectious agents and subsequent testing for antibiotic sensitivity and resistance. This process requires lengthy cultivation periods (days) and is not applicable for certain bacterial infections, or for most viral infections. Nucleic acid amplification-based tests (NAAT) for direct pathogen detection are showing considerable promise. Their advantages include high sensitivity and the simultaneous detection of multiple pathogens. Consequently, these tests are increasingly used in hospital and laboratory settings $[19,20]$. However, NAAT protocols exhibit varying degrees of sensitivity and specificity when identifying specific pathogens and antibiotic resistance traits. In addition, NAAT diagnostic technologies usually require direct sampling of the pathogen. Such sampling is often not easily feasible if the infection site is not easily accessible (e.g., sinusitis, middle-ear infection and bronchitis) or the site of infection is unknown (e.g., fever of unknown origin).

\section{- Reduced clinical utility due to colonizers}

Moreover, currently available diagnostic approaches often suffer from reduced clinical utility because they do not distinguish between pathogenic strains of microorganisms and potential colonizers, which can be present as part of the natural microbiota without causing an infection [21-24]. For example, Rhedin and colleagues recently tested the clinical utility of quantitative PCR (qPCR) for common viruses in acute respiratory illness [24]. The authors concluded that qPCR detection of several respiratory viruses including rhinovirus, enterovirus and coronavirus should be interpreted with caution due to high detection rates in asymptomatic children. Other studies reached similar conclusions after analyzing the detection rates of different bacterial strains in asymptomatic patients [25,26].

The described limitations of current diagnostic procedures lead physicians to either overprescribe ('just-in-case’) or underprescribe ('watchful waiting') antibiotics, both of which can adversely impact patient care and health economics. Therefore, there is a clear need for novel solutions that will empower physicians to make early, evidence-based antibiotic treatment decisions, in order to improve patient care, reduce adverse events and limit the spread of antimicrobial resistance.

\section{A multifaceted 'omics' approach for reducing antibiotic resistance}

Meeting the challenges of antibiotic resistance requires adopting an integrative approach that expands the antimicrobial arsenal on the one hand and reduces antibiotic consumption on the other [11,27-28]. New antibiotic drugs are desired, but will likely provide only a temporary solution in light of the inherent ability of microbes to develop new resistances by a variety of physiological mechanisms, including the exchange of transferable genetic elements, uptake of DNA by transformation and transduction and DNA mutation. Moreover, only a handful of new antibiotics are likely to reach the market in the near future due to the 'broken pipeline' associated with their development [29]. Several factors have undermined the economic incentives for the development of new antibiotics in recent years, including: the significant financial commitment required for developing new drugs (including research, development and regulatory approval costs); the use of antibiotics for brief periods of time, usually on a 'need-only' basis; the restricted use of new and costlier antibiotics for more severe cases as a 'last-resort' option; and the higher economic incentives for developing medications for the management of chronic conditions, which originate from their continuous consumption for long periods of time (months or years). Recently, in trying to fix this 'broken pipeline', several governmental initiatives in the USA and Europe have been launched, in order to provide financial incentives for the development of new, innovative antibiotic drugs [11,30-31].

Complementary to the development of new drugs is the need for a significant reduction 
in antibiotic consumption. This goal may be achieved by increasing public awareness of antibiotic misuse and its consequences, as well as by adopting new diagnostic approaches to bridge the current diagnostic gap. However, though recent antimicrobial stewardship programs have gained some success in reducing overall antibiotic consumption in several countries across the globe [32], further improvements in global antibiotic prescribing practices are still required $[1,33]$.

New technologies are constantly emerging, providing the scientific and medical communities with powerful tools to improve the diagnosis of infectious diseases. Advances in the analysis of pathogens and the host immune response to infection in a broad and sensitive manner have led to a deeper understanding of complex host-pathogen-treatment interactions [28]. Technologies such as next-generation sequencing provide snapshots of the patient's entire transcriptome in response to an infection or treatment, as well as enable the genetic analysis of the patient's natural microbiota (microbiome). These technologies also facilitate the search for novel genetic variation markers (single nucleotide polymorphisms [SNPs]) that may predispose individuals to specific infections and disease progression. Most importantly, advances in bioinformatics algorithms and 'big-data' analysis enable the integration of clinically relevant information such as host genetics, microbiota, response to treatment and data on the disease-causing agent. Mining this integrated data to generate treatment algorithms, coupled with the development of intuitive web-based interfaces, will be a major step forward in the management of infectious disease patients. Not least in the ongoing battle against antimicrobial resistance.

\section{The TAILORED-Treatment research program}

The EU is investing public funds in a range of multidisciplinary scientific programs that harness recent technological advances for addressing the challenges of antimicrobial resistance. One of the recently funded projects is the 4-year 'TAILORED-Treatment' research program, which focuses on the diagnosis of infectious disease etiologies for guiding antibiotic treatment in patients with respiratory tract infections and/or sepsis [34]. The project combines state-of-the-art omics-based techniques and data with multivariate analysis methods and newly developed bioinformatics software with the goal of translating novel host-pathogen insights into treatment decision support algorithms. At the heart of the project is a large multicenter prospective clinical study enrolling 1200 adult and pediatric patients, where patient and microbe-related information is being collected (Figure 2). The data are being investigated for significant associations between multiple biological and clinical parameters (Figure 3). New scientific discoveries generated by such omics-based approach will serve as a foundation for novel diagnostic solutions and for new web-based decision support systems for the physician of the future. The 'omics' approach of the TAILORED-Treatment program for personalizing antimicrobial treatment is further described below.

\section{- Host genomic analysis}

SNPs are the most common type of genetic variation found among individuals, with approximately 10 million SNPs in the human genome [36]. Each SNP represents a difference in a single nucleotide at a single position in the genome, which can occur in noncoding, regulatory or coding regions, and thus can affect the function of specific genes. Thousands of SNPs have already been shown to have a robust association with hundreds of different traits and diseases [37]. Importantly, recent genome-wide studies have reported novel associations between common polymorphisms and susceptibility to several major infectious diseases of humans [38]. For example, genetic polymorphisms were implicated in the susceptibility, severity and outcome of meningococcal disease [39,40]; polymorphisms in the Toll-like receptor 2 and the anti-infective cytokine IL-17A gene increase the risk of serious Gram-positive infections [41], and a specific set of host genetic polymorphisms was recently suggested to be the predominant determinant for Staphylococcus aureus persistent nasal carriage [42] .

Genetic polymorphism can also determine the response to treatment [36]. Multiple genome-wide association studies in different populations have reported strong associations between IL-28B polymorphism and the response to hepatitis $C$ virus treatment [43-46]. Other studies have found associations between genetic variation in the HLA-B region and hypersensitivity reactions to HIV therapy [47-49], and suggested genotypic testing as a simple predictive screening tool for hypersensitivity reactions to specific anti-HIV drugs [50,51]. Host genome analysis is being performed in infectious 


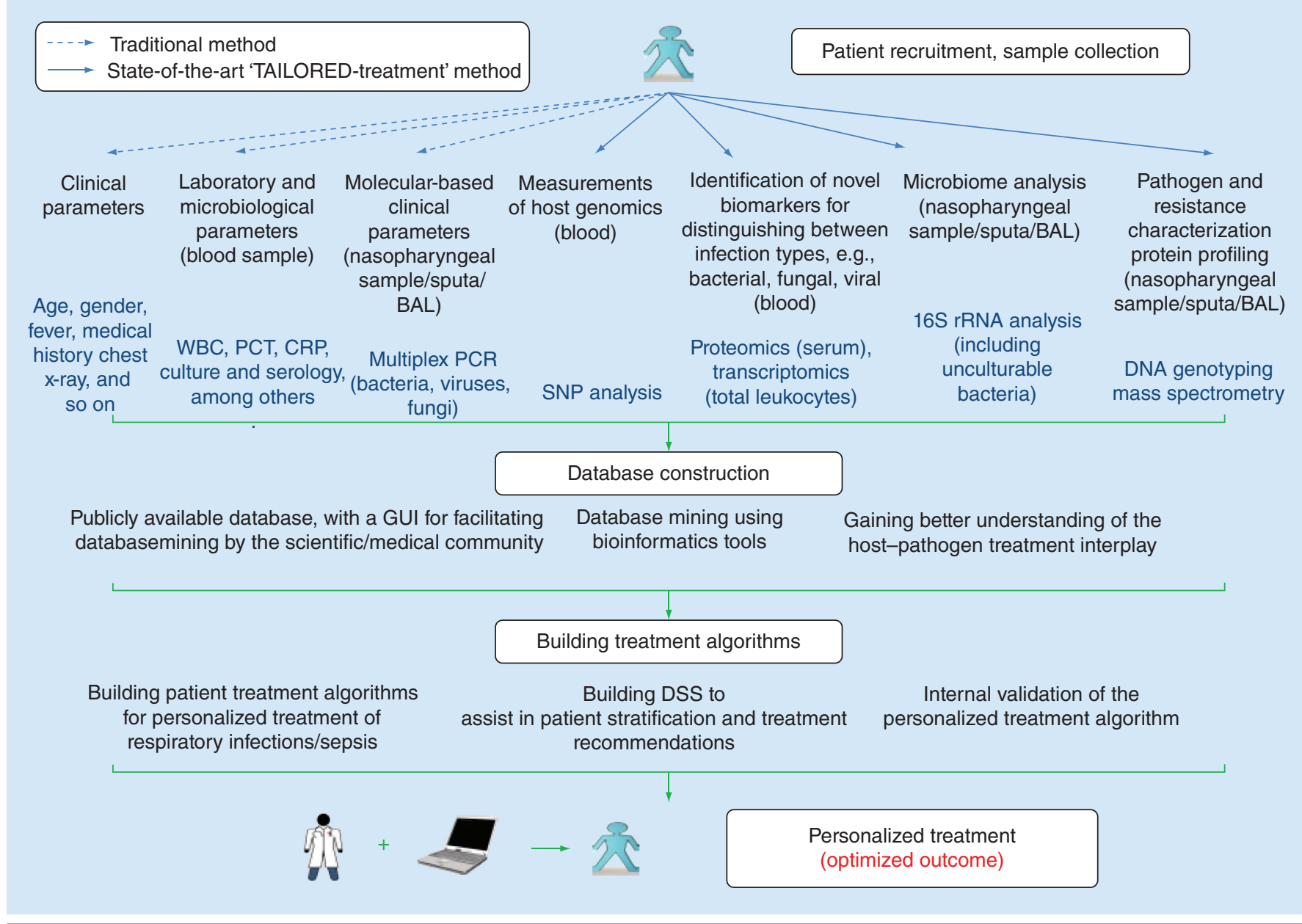

Figure 2. Multifaceted approach adopted by the TAILORED-Treatment consortium to help personalize antimicrobial prescribing practices to individual patients. Traditional and state-of-the-art omics-based techniques are being utilized to perform comprehensive patient and pathogen analyses that combine clinical data, proteomics, transcriptomics, microbiome analysis, genetics and bioinformatics. The collected data are used to identify novel host-pathogen-treatment dynamics and generate a web-based predictive treatment algorithm for use by physicians.

BAL: Bronchoalveolar lavage; CRP: C-reactive protein; DSS: Decision support systems; GUI: Graphical user interface; PCT: Procalcitonin; SNP: Single nucleotide polymorphism; WBC: White blood cell.

Reproduced with permission from [35].

disease patients in order to identify novel SNPs potentially associated with predisposition to specific types of infections, disease progression and response to treatment (pharmacogenomics).

\section{- Host transcriptomic \& proteomic response to infection}

Fashioned by evolution, our immune system has evolved for millions of years to distinguish between bacterial, fungal and viral pathogens. Deciphering the host response to these different types of infections can reveal valuable diagnostic biomarkers for guiding clinical treatment decisions. Importantly, this approach addresses several challenges posed by current microbiological tests by enabling: diagnosis even in cases of not easily accessible or unknown infection sites (e.g., lower respiratory tract infection, fever without a known source in children); rapid measurement of soluble host proteins (within minutes) on hospital-deployed automated immunoassay machines and point-of-care devices; and diagnosis that is not affected by the presence of potential colonizers that comprise our natural microbiota. Based on this, a paradigm shift for diagnosing infections has been gaining ground in recent years: instead of targeting potential infectious pathogens, diagnosis 


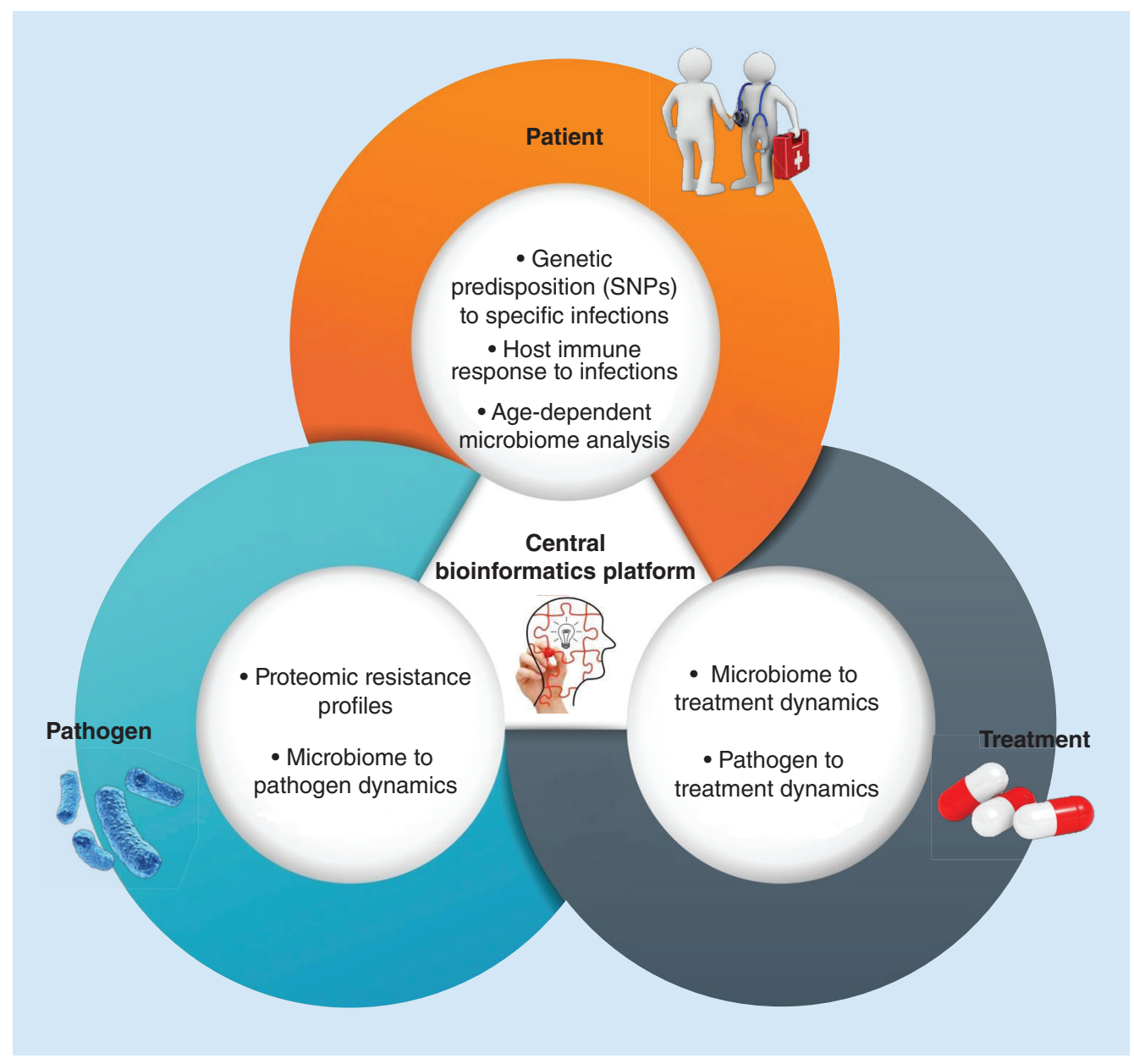

Figure 3. New insights to be gained using a multifaceted omics-based approach for studying patient-pathogen-treatment interactions. Omics-based surveys of the host transcriptome, proteome, genome and microbiome within a large cohort of patients will generate a substantial amount of novel data. The newly collected data are stored and mined using a dedicated bioinformatics platform, enabling efficient statistical analysis and the identification of significant associations between the different data sets.

SNP: Single nucleotide polymorphism.

could be based on the host immune response to infection [52,53].

Traditional high-throughput RNA measurements have identified promising novel hostRNA biomarkers [52-58]. The pioneering works of Ramilo [57] and Zaas [56] have demonstrated, in small cohorts of human subjects, the potential of gene-expression patterns to discriminate between patients with different etiologies. However, applying host RNA-based signatures in the clinical setting requires discovery and validation of RNA signatures in large cohorts of human patients presenting with a wide range of pathogens. Additionally, most RNA biomarker discovery efforts have thus far been focused on gene level exploration (i.e., mRNA) [54-57], although up to $70 \%$ of the human genome is transcribed into RNA that does not serve as templates for proteins [59]. Next-generation sequencing technologies and novel human transcriptome microarrays allow high-throughput, quantitative surveys of the transcriptome, capturing a broader range of gene expression changes in response to external conditions and enabling the detection of novel transcripts including mRNAs, noncoding RNAs and small RNAs [60,61]. Although these technologies were introduced only several years ago, they have already triggered numerous groundbreaking discoveries and ignited a revolution in genomic and transcriptomic science $[60,61]$.

The TAILORED-Treatment project is using biochemical and advanced high-throughput 
technologies to screen both the host proteome and host transcriptome in search of novel biomarkers that distinguish between viral, bacterial and fungal infections, both in children and adults. Comprehensive transcriptome analysis is being performed to explore the immune arm of the noncoding RNA, being based on recent evidence that noncoding RNAs may be valuable building blocks of the host response to infection [62]. Machine learning schemes are being used to generate multiparametric signatures combining different biomarkers in order to overcome the challenge of patient-to-patient variability.

\section{- Microbiota/microbiome analysis}

A microbiome is defined as the collective genomes of various microorganism communities (microbiota) that inhabit and share our body space [63]. This includes the microbiota found in the gut, mouth, nostril and vagina, as well as the microbiota present on the skin [64]. Approximately $90 \%$ of the cells in and on the human body are microbial cells, which routinely contribute to tissue and organ function [63]. The microbiota composition may correlate with an individual's genotype, diet and overall health status. For example, a 'Western' diet comprising high intakes of protein and fat may generate a different gut microbiota/ microbiome when compared with an agrarian plant-rich diet [65]. Thus, studying the different characteristics of microbial communities can provide valuable information on human health and disease predisposition [66,67]. In 2007, the NIH launched the Human Microbiome Project, aimed at creating a reference cata$\log$ of microbial DNA for building data sets that may provide insight into how an individual's microbiome signature relates to his/ her health and disease status [68]. Thus far, Human Microbiome Project research centers have generated over 14 terabytes of new microbiome sequence data, which are available to the global community of microbiologists [69]. Using these new data, studies have associated the human microbiome with a wide range of pathologies, including those of the gastrointestinal tract, respiratory tract, skin and nervous system $[66,67]$. Recent reports also indicate that the microbiota composition is a major predictive factor of the type and robustness of the host mucosal immune response [70]. Finally, it has been shown that antibiotic treatment affects both the disease-causing agent and the microbial ecology, thereby affecting host metabolism [71].

In light of the emerging importance of the microbiota/microbiome in health and disease, and the new technologies that enable its broad analysis, a patient's microbiota/microbiome should be considered a critical component when developing personalized antimicrobial treatment schemes. The TAILOREDTreatment project is mapping the respiratory (nasal) microbiota in children and adults suffering from respiratory or sepsis-related disease in order to discover new host-microbe interactions related to age, gender and treatment. Age-related differences in the human microbiome have already been described [72] Microbiota maps are also being integrated with relevant clinical data in order to assess how a patient's natural microbiota affects pathogenicity levels, disease progression and overall health outcome.

\section{- Rapid profiling of pathogens}

The TAILORED-Treatment program is also seeking to develop and improve techniques for rapid pathogen identification and antimicrobial resistance profiling. Increasingly, microbiology laboratories are using mass spectrometry to study microbial pathogens and to identify antibiotic resistances. However, validation of this technique on a diverse spectrum of clinical samples is a prerequisite for its widespread use. Moreover, such procedures currently require that infectious microorganisms first be cultured, which imposes significant time constraints on patient treatment regimens. The development and integration of a mass spectrometry-based method that can rapidly identify and characterize bacterial isolates directly from clinical specimens (i.e., without the prior cultivation of the infectious microorganisms) would enable 'real-time' informed decisions to be made regarding whether to administer antibiotic therapy to a patient, and which class of antibiotics to use.

A mass spectrometry proteomics-based approach is being further developed in order to reduce the time from sample collection to full pathogen profiling, as another step toward the adoption of this technique in different clinical settings. Such diagnostic tools will provide answers to physicians within $5 \mathrm{~h}$ from the time of sample collection, compared with approximately 
$48 \mathrm{~h}$ using current gold-standard procedures for identifying pathogens and their antibiotic resistance. To achieve this, technologies such as lipid-based protein immobilization are being utilized, whereby intact bacterial cells are bound, via membrane-gold interactions, within a flow cell [73]. The bound cells are subjected to enzymatic digestion for the generation of peptides, which are subsequently identified, using LC-MS. Following database matching, strain-specific peptides are used for subspecies-level discrimination. This method has been shown to enable reliable typing and identification of closely related strains of the same bacterial species $[73,74]$.

\section{- Systems level analyses}

Omics-based surveys of the host genome, transcriptome, proteome and microbiome within a large cohort of patients will generate a substantial amount of different types of data. One of the biggest challenges toward gaining new insights into host-pathogen-treatment dynamics is the integrative analysis of different layers of data, required for identifying significant associations between the various data sets. As a first step, classical clustering (e.g., K-means [75] or different kinds of hierarchical clustering) and feature selection algorithms (e.g., Relieff or Logo [76]) are required to identify key variables, to weight the importance of these variables as markers and to test the predictive power of these markers. Using these algorithms to analyze diverse data often requires their customization or the development of new software tools that can enable the joint analysis of different types of data (e.g., categorical and numerical variables). The next step is to apply a range of tools to process the integrated data and extract systemic associations. For example, association rule learning is a popular and well-researched method for discovering interesting relations between variables in large databases [77]. Additionally, decision trees (e.g., C4.5 [78]) can provide an intuitive view of the behavior of the data, while functional enrichment tools (e.g., integrating gene ontology annotations) can allow the integration of expert knowledge within the analytical results. More sophisticated dimensionality reduction algorithms (e.g., isometric feature mapping [ISOMAP] [79]) are necessary to enable the modeling of complex manifolds comprising data from different sources, whose behavior would be impossible to reproduce using simpler algorithmic methods. Finally, in order to simplify the direct and rapid extraction of information the tools described above should be integrated with associated databases in a common framework. The advantages of this combination include the possibility to set up preconfigured pipelines that are targeted to the analysis of different types of data, the reliable definition of costumed sub-data sets and the establishment of target variables, that is, the definition of the question to be answered by the system.

\section{- Web-based decision support system}

The knowledge gained using omics-based data collection and analysis is being used to develop new personalized treatment algorithms. Such algorithms will help physicians tailor antimicrobial therapies to the specific and evidencebased requirements of children and adults presenting with respiratory infections and sepsis. Personalized treatment algorithms are being integrated into a simple, web-based interface that will be made available to physicians, allowing them to make predictive clinical decisions, including the correct targeting of antibiotic treatment to the actual personal needs of the patient.

\section{Conclusion}

The problem of increasing antibiotic resistance is a global phenomenon that is now being appreciated by governments and citizens alike. No single solution is able to avert the continuing dissemination of worldwide multiresistant bacterial strains. Instead, a consolidated and coordinated global effort is required, which includes the development of more rapid and accurate diagnostics for bacterial infections. With this in mind, the TAILORED-Treatment concept takes a step towards the near future, where omics data will be readily available for consultation by physicians and (together with clinical data) will help provide essential information for the rapid and accurate diagnosis of bacterial infections.

\section{Future perspective}

Technological advances in the current postgenomic era will continue to generate a wealth of data at both the patient and pathogen levels. This is due to the publication of a growing number of complete genome sequences for a large range of clinically relevant pathogenic microorganisms, as well as the publication of complete human genomes from subjects representing a wide range of ages, gender and races [80]. Novel 
desktop-size machines can now rapidly sequence a whole human genome at a cost approaching US $\$ 1000$, compared with over US $\$ 2$ billion (the cost of the human genome project a decade ago). Sequencing devices are becoming smaller and cheaper, making the dream of using personal genomic and transcriptomic data in routine visits to the doctor's office a tangible reality. Concurrently, the information revolution of recent years has allowed the clinical community to address common practice challenges by sharing tools, information, scientific publications, and experience. Additionally, affordable smartphones are becoming an integral part of medical practice, with a variety of applications that literally bring healthcare to the palm of the clinician's and patient's hands. Real-time monitoring of patient vital signs together with the constant delivery of healthcare information to clinicians empowers patients to control their chronic conditions and maintain their normal daily routine. This approach is expected to be significantly enhanced by the introduction of new wearable health devices, which will increase the variety of vital signs that can be constantly monitored [81]. These technological advancements are expected to facilitate the integration of personalized data into routine clinical care. We envision a future where physicians will use machine learningbased decision support systems, which integrate patient personal data with host and pathogen diagnostic biomarkers (Figure 4), to diagnose and treat patients. Patient diagnosis will combine traditional clinical parameters with omicsbased personal data, including: the genetic predisposition to certain types of infections; the response to different classes of drugs; and the dynamics of the microbiota/microbiome in response to treatment. Computerized integration of these data will mean that real-time recommendations can be made regarding the optimal treatment regimen for each patient, as well as a prediction of patient prognosis and health outcome. The challenge for the future will be how to best interpret and efficiently act on this wealth of data. The timely creation of validated algorithms and simplified user interfaces will help establish a new range of 'gold standards' in personalized clinical treatment, ultimately informing and advising both clinicians and patients on the best treatment options available. The extensive use of such multifaceted algorithms for the management of infectious disease patients will not only improve patient

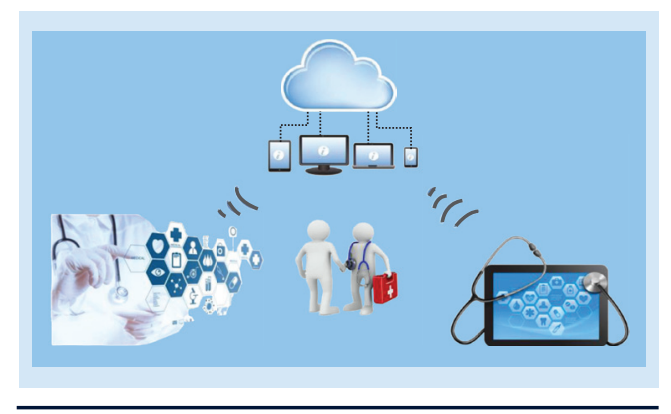

Figure 4. The future of personalized infectious disease therapy. Cloud-based computational platforms will be integrated with patient-based omics information such as genomics, transcriptomics, proteomics, microbiome and drug metabolism kinetics to generate accurate physician-friendly algorithms. Combining accessibility to personal data and algorithms with point-of-care diagnostic tests has the potential to transform the management of infectious disease.

care but also help reduce antibiotic consumption, ultimately limiting the spread of antibiotic resistance.

\section{Acknowledgements}

The authors would like to acknowledge the following coworkers on the TAILORED-Treatment project, who together with the authors are helping to realize the project goals: Vincent Collins, Eva González, Hans Hoogeveen, Chantal van Houten, Erik Kristiansson, Anders Karlsson, Roger Karlsson, Robert Kraaij, Julio Font Perez and Andrew Stubbs. The authors would also like to thank Andriy Nemchenko for assistance with the graphics and Iris Kulbatski for scientific comments and reviewing the manuscript.

\section{Financial \& competing interests disclosure}

This publication has received funding from the EU's Seventh Framework Programme FP7 under REA grant agreement No. HEALTH-F3-602860-2013 (TAILOREDTreatment; www.tailored-treatment.eu). The authors have no other relevant affiliations or financial involvement with any organization or entity with a financial interest in or financial conflict with the subject matter or materials discussed in the manuscript apart from those disclosed.

No writing assistance was utilized in the production of this manuscript.

\section{Open access}

This work is licensed under the Creative Commons Attribution-NonCommercial 3.0 Unported License. To view a copy of this license, visit http://creativecommons.org/ licenses/by-nc-nd/3.0/ 


\section{EXECUTIVE SUMMARY}

The antibiotic resistance crisis

- Despite their immense contribution to human health, up to $50 \%$ of all the antibiotics prescribed for people are not needed or are not optimally effective as prescribed.

- One of the most alarming consequences of antibiotic overuse is the emergence and spread of multidrug-resistant bacteria.

- Resistant bacteria infect over 2 million people annually in the USA and over 400,000 people in the EU, resulting in thousands of deaths.

- Antibiotic-resistant infections cost the US healthcare system an estimated US\$20 billion annually, with an additional estimated US\$35 billion in lost productivity.

\section{A diagnostic gap}

- Among the factors underlying antibiotic misuse is the challenge of accurately distinguishing between bacterial infections (which warrant antibiotic therapy) and viral infections (for which antibiotic treatment is generally not required).

- This diagnostic gap is driven by the inability of current diagnostic tools to provide rapid and accurate information about the etiology of an infection.

- Reaching a definite diagnosis is complicated by not easily accessible or unknown infection sites, the difficulty to distinguish between pathogenic and commensal bacteria and the prolonged time required to achieve a definitive result.

\section{A multifaceted 'omics' approach for reducing antibiotic resistance}

- Meeting the challenges of antibiotic resistance will include the development of new drugs and innovative strategies for improving diagnosis and treatment of infections.

- Gaining deeper understanding of complex host-pathogen treatment interactions through new omics-based technologies offers a unique opportunity for the development of personalized diagnosis and treatment schemes.

- This approach requires adopting an integrative approach to clinical diagnosis and disease prognosis prediction that brings together different disciplines from the medical, computational and life sciences.

The TAILORED-Treatment research program

- The EU recently funded a multidisciplinary research program (TAILORED-Treatment) to improve the understanding of host-pathogen treatment dynamics in respiratory and septic patients.

- The project combines clinical data, proteomics, transcriptomics, microbiome analysis, genetics and bioinformatics to develop novel personalized treatment algorithms.

- These algorithms will be integrated into a web-based decision support system that will be made available to physicians.

- Evidence-based decision support systems will enable faster, better-informed antibiotic treatment decisions, thereby limiting the emergence and spread of antibiotic resistance.

\section{References}

Papers of special note have been highlighted as: - of interest

1 CDC. Threat Report 2013. Antimicrobial Resistance.

www.cdc.gov

- The most up-to-date and comprehensive report describing the antibiotic resistance threats in the USA.

2 CDC - About Antimicrobial Resistance. www.cdc.gov/drugresistance/about.html
3 Bartlett JG. Clinical practice. Antibioticassociated diarrhea. N. Engl. J. Med. 346(5), 334-339 (2002).

4 Craig JC, Williams GJ, Jones $\mathrm{M}$ et al. The accuracy of clinical symptoms and signs for the diagnosis of serious bacterial infection in young febrile children: prospective cohort study of 15,781 febrile illnesses (2010). www.ncbi.nlm.nih.gov

5 Dedier J, Singer DE, Chang Y, Moore M, Atlas SJ. Processes of care, illness severity, and outcomes in the management of communityacquired pneumonia at academic hospitals. Arch. Intern. Med. 161(17), 2099-2104 (2001).

6 Caterino JM, Hiestand BC, Martin DR. Quality of care in elder emergency department patients with pneumonia: a prospective cohort study. BMC Emerg. Med. 8, 6 (2008).

7 Houck PM. Timing of antibiotic administration and outcomes for medicare patients hospitalized with community- 
acquired pneumonia. Arch. Intern. Med. 164(6), 637-644 (2004).

8 Zwart S, Sachs AP, Ruijs GJ, Gubbels JW, Hoes AW, de Melker RA. Penicillin for acute sore throat: randomised double blind trial of seven days versus three days treatment or placebo in adults. BMJ 320 (7228), 150-154 (2000).

9 Little P. Delayed prescribing of antibiotics for upper respiratory tract infection. $B M J$ 331(7512), 301-302 (2005).

10 Spiro DM, Tay K-Y, Arnold DH, Dziura JD, Baker MD, Shapiro ED. Wait-and-see prescription for the treatment of acute otitis media: a randomized controlled trial. J. Am. Med. Assoc. 296(10), 1235-1241 (2006).

11 Fauci AS, Marston HD. The perpetual challenge of antimicrobial resistance. JAMA 311(18), 1853-1854 (2014).

12 Armstrong GL, Conn LA, Pinner RW. Trends in infectious disease mortality in the United States during the 20th century. J. Am. Med. Assoc. 281(1), 61-66 (1999).

13 Hollis A, Ahmed Z. Preserving antibiotics, rationally. N. Engl. J. Med. 369 (26), 2474-2476 (2013).

14 CDC. Get smart: fast facts about antibiotic resistance. www.cdc.gov

15 European Surveillance of Antimicrobial Consumption Network (ESAC-Net). www.ecdc.europa.eu

16 WHO Europe. Data and statistics. www.euro.who.int

17 WHO. Antimicrobial resistance. www.who.int

18 Arias CA, Murray BE. Antibiotic-resistant bugs in the 21st century - a clinical super-challenge. N. Engl. J. Med. 360 (5), 439-443 (2009).

19 Niemz A, Ferguson TM, Boyle DS. Point-of-care nucleic acid testing for infectious diseases. Trends Biotechnol. 29(5), 240-250 (2011).

20 Craw P, Balachandran W. Isothermal nucleic acid amplification technologies for point-ofcare diagnostics: a critical review. Lab. Chip. 12(14), 2469-2486 (2012).

21 Kim KH, Shin JH, Kim SY. The clinical significance of nasopharyngeal carriages in immunocompromised children as assessed. Korean J. Hematol. 44(4), 220-226 (2009).

22 Shin JH, Han HY, Kim SY. Detection of nasopharyngeal carriages in children by multiplex reverse transcriptase-polymerase chain reaction. Korean J. Pediatr. 52(12), 1358 (2009).
23 Jung CL, Lee MA, Chung WS. Clinical evaluation of the multiplex PCR Assay for the detection of bacterial pathogens in respiratory specimens from patients with pneumonia. Korean J. Clin. Microbiol. 13(1), 1358-1363 (2010).

24 Rhedin S, Lindstrand A, Rotzén-Östlund M et al. Clinical utility of PCR for common viruses in acute respiratory illness. Pediatrics 133(3), e538-e545 (2014).

- Provides a comprehensive evaluation of qPCR tests for common viruses in acute respiratory illness.

25 Bogaert D, De Groot R, Hermans PWM. Streptococcus pneumoniae colonisation: the key to pneumococcal disease. Lancet Infect. Dis. 4(3), 144-154 (2004).

26 Spuesens EBM, Fraaij PLA, Visser EG et al. Carriage of Mycoplasma pneumoniae in the upper respiratory tract of symptomatic and asymptomatic children: An observational study. PLoS Med. 10(5), e1001444 (2013).

27 Laxminarayan R, Duse A, Wattal C et al. Antibiotic resistance-the need for global solutions. Lancet Infect. Dis. 13(12), 1057-1098 (2013).

28 Aderem A, Adkins JN, Ansong C et al. A systems biology approach to infectious disease research: innovating the pathogen-host research paradigm. mBio 2(1), e00325-e00310 (2011).

29 Cooper MA, Shlaes D. Fix the antibiotics pipeline. Nature 472(7341), 32 (2011).

30 Press Announcements - new FDA task force will support innovation in antibacterial drug development. www.fda.gov

31 Kirby T. Europe to boost development of new antimicrobial drugs. Lancet 379(9833), 2229-2230 (2012).

32 IMS Institute. Responsible Use of Medicines Report 2012.

www.imshealth.com

33 Too Many Antibiotics! Patients and Prescribers Speak Up. www.medscape.com

34 Tailored-Treatment. www.tailored-treatment.eu

35 Tailored-Treatment. Goals and concept. www.tailored-treatment.eu/?page_id=7

36 Laing RE, Hess P, Shen Y, Wang J, Hu SX. The role and impact of SNPs in pharmacogenomics and personalized medicine. Curr. Drug Metab. 12(5), 460-486 (2011).

37 Hofker M, Fu J, Wijmenga C. The genome revolution and its role in understanding complex diseases. Biochim. Biophys. Acta. 1842(10), $1889-1895$ (2014).

38 Chapman SJ, Hill AVS. Human genetic susceptibility to infectious disease. Nat. Rev. Genet. 13(3), 175-188 (2012).

39 Emonts M, Hazelzet JA, de Groot R, Hermans PWM. Host genetic determinants of Neisseria meningitidis infections. Lancet Infect. Dis. 3(9), 565-577 (2003).

40 Brouwer MC, de Gans J, Heckenberg SGB, Zwinderman AH, van der Poll T, van de Beek D. Host genetic susceptibility to pneumococcal and meningococcal disease: a systematic review and meta-analysis. Lancet Infect. Dis. 9(1), 31-44 (2009).

41 Boyd JH, Russell JA, Fjell CD. The meta-genome of sepsis: host genetics, pathogens and the acute immune response. J. Innate Immun. 6(3), 272-283 (2014).

42 Ruimy R, Angebault C, Djossou F et al. Are host genetics the predominant determinant of persistent nasal Staphylococcus aureus carriage in humans? J. Infect. Dis. 202(6), 924-934 (2010).

43 Ge D, Fellay J, Thompson AJ et al. Genetic variation in IL28B predicts hepatitis C treatment-induced viral clearance. Nature 461(7262), 399-401 (2009).

44 Rauch A, Kutalik Z, Descombes P et al. Genetic variation in IL28B is associated with chronic hepatitis $\mathrm{C}$ and treatment failure: a genomewide association study. Gastroenterology 138(4), 1338-1345, 1345.e1-7 (2010).

45 Tanaka Y, Nishida N, Sugiyama M et al. Genome-wide association of IL28B with response to pegylated interferon-alpha and ribavirin therapy for chronic hepatitis C. Nat. Genet. 41(10), 1105-1109 (2009).

46 Ochi H, Maekawa T, Abe H et al. IL-28B predicts response to chronic hepatitis $\mathrm{C}$ therapy - fine-mapping and replication study in Asian populations. J. Gen. Virol. 92(Pt 5), 1071-1081 (2011).

47 Hetherington S, Hughes AR, Mosteller M et al. Genetic variations in HLA-B region and hypersensitivity reactions to abacavir. Lancet 359(9312), 1121-1122 (2002).

48 Mallal S, Nolan D, Witt C et al. Association between presence of HLA-B*5701, HLADR7, and HLA-DQ3 and hypersensitivity to HIV-1 reverse-transcriptase inhibitor abacavir. Lancet 359(9308), 727-732 (2002).

49 Martin AM, Nolan D, Gaudieri S et al. Predisposition to abacavir hypersensitivity conferred by HLA-B*5701 and a haplotypic Hsp70-Hom variant. Proc. Natl Acad. Sci. USA 101(12), 4180-4185 (2004).

50 Colombo S, Rauch A, Rotger $\mathrm{M}$ et al. The HCP5 single-nucleotide polymorphism: 
a simple screening tool for prediction of hypersensitivity reaction to abacavir. J. Infect. Dis. 198(6), 864-867 (2008).

51 Young B, Squires K, Patel P et al. First large, multicenter, open-label study utilizing HLA-B*5701 screening for abacavir hypersensitivity in North America. AIDS Lond. Engl. 22(13), 1673-1675 (2008).

52 Mejias A, Suarez NM, Ramilo O. Detecting specific infections in children through host responses: a paradigm shift. Curr. Opin. Infect. Dis. 27(3), 228-235 (2014).

53 Ramilo O, Mejías A. Shifting the paradigm: host gene signatures for diagnosis of infectious diseases. Cell Host Microbe 6(3), 199-200 (2009).

- These two papers describe the paradigm shift for diagnosing infections using the host differential immune response following the groundbreaking discoveries of their and other research groups.

54 Huang Y, Zaas AK, Rao A et al. Temporal dynamics of host molecular responses differentiate symptomatic and asymptomatic influenza a infection. PLoS Genet. 7(8), e1002234 (2011).

55 Woods CW, McClain MT, Chen M et al. A host transcriptional signature for presymptomatic detection of infection in humans exposed to influenza $\mathrm{H} 1 \mathrm{~N} 1$ or H3N2. PloS ONE 8(1), e52198 (2013).

56 Zaas AK, Chen M, Varkey J et al. Gene expression signatures diagnose influenza and other symptomatic respiratory viral infections in humans. Cell Host Microbe 6(3), 207-217 (2009).

57 Ramilo O, Allman W, Chung W et al. Gene expression patterns in blood leukocytes discriminate patients with acute infections. Blood 109(5), 2066-2077 (2007).

58 Mahajan P, Ramilo O, Kuppermann N. The future possibilities of diagnostic testing for the evaluation of febrile infants. JAMA Pediatr. 167(10), 888-898 (2013).
59 Gutschner T, Diederichs S. The hallmarks of cancer: a long non-coding RNA point of view. RNA Biol. 9(6), 703-719 (2012).

60 Kato K. Impact of the next generation DNA sequencers. Int. J. Clin. Exp. Med. 2(2), 193-202 (2009).

61 Wang Z, Gerstein M, Snyder M. RNA-Seq: a revolutionary tool for transcriptomics. Nat. Rev. Genet. 10(1), 57-63 (2009).

62 Peng X, Gralinski L, Armour CD et al. Unique signatures of long noncoding RNA expression in response to virus infection and altered innate immune signaling. mBio 1(5) e00206-10 (2010).

63 Gill SR, Pop M, Deboy RT et al. Metagenomic analysis of the human distal gut microbiome. Science 312 (5778), 1355-1359 (2006).

64 Cho I, Blaser MJ. The human microbiome: at the interface of health and disease. Nat. Rev. Genet. 13(4), 260-270 (2012).

- A comprehensive review that describes the role the human microbiome play in health and disease.

65 Albenberg LG, Wu GD. Diet and the intestinal microbiome: associations, functions, and implications for health and disease. Gastroenterology 146(6), 1564-1572 (2014).

66 Pflughoeft KJ, Versalovic J. Human microbiome in health and disease. Annu. Rev. Pathol. 7, 99-122 (2012).

67 Madupu R, Szpakowski S, Nelson KE. Microbiome in human health and disease. Sci. Prog. 96(Pt 2), 153-170 (2013).

68 Human Microbiome Project DACC - home. www.hmpdacc.org/

69 Human Microbiome Project - home. http://commonfund.nih.gov/hmp/index

70 Honda K, Littman DR. The microbiome in infectious disease and inflammation. Annu. Rev. Immunol. 30(1), 759-795 (2012)

71 Pérez-Cobas AE, Gosalbes MJ, Friedrichs A et al. Gut microbiota disturbance during antibiotic therapy: a multi-omic approach. Gut. 62(11), 1591-1601 (2012).
72 Lan Y, Kriete A, Rosen GL. Selecting age-related functional characteristics in the human gut microbiome. Microbiome 1(1), 2 (2013).

73 Karlsson R, Davidson M, Svensson-Stadler L et al. Strain-level typing and identification of bacteria using mass spectrometry-based proteomics. J. Proteome Res. 11(5), 2710-2720 (2012).

74 Chooneea D, Karlsson R, Encheva V, Arnold C, Appleton H, Shah H. Elucidation of the outer membrane proteome of Salmonella enterica serovar Typhimurium utilising a lipid-based protein immobilization technique. $B M C$ Microbiol. 10, 44 (2010).

75 Lloyd S. Least squares quantization in PCM. IEEE Trans. Inf. Theory 28(2), 129-137 (1982).

76 Cai H, Ruan P, Ng M, Akutsu T. Feature weight estimation for gene selection: a local hyperlinear learning approach. $B M C$ Bioinformatics 15(1), 70 (2014).

77 Srikant R, Agrawal R. Mining generalized association rules. Future Gener. Comput. Syst. 13(2-3), 161-180 (1997).

78 Salzberg SL. C4.5: Programs for Machine Learning by J. Ross Quinlan. Morgan Kaufmann Publishers, Inc., 1993. Mach. Learn. 16(3), 235-240 (1994).

79 Tenenbaum JB, de Silva V, Langford JC. A global geometric framework for nonlinear dimensionality reduction. Science 290(5500), 2319-2323 (2000).

80 Durmu Tekir SD, Ülgen KÖ. Systems biology of pathogen-host interaction: networks of protein-protein interaction within pathogens and pathogen-human interactions in the post-genomic era. Biotechnol. J. 8(1), 85-96 (2013).

81 InformationWeek. 10 wearable health tech devices to watch. www.informationweek.com 